\title{
COMMENTARY
}

\section{Optimal glycemic control in neurocritical care patients}

\author{
Federico Bilotta* ${ }^{* \dagger}$ and Giovanni Rosa ${ }^{\dagger}$ \\ See related research by Kramer et al., http://ccforum.com/content/16/5/R203
}

\begin{abstract}
Currently, the major issue in glycemic control in neurocritical care patients is that tight glycemic control (target range of 80 to $110 \mathrm{mg} / \mathrm{dL}$ ) using intensive insulin therapy is associated with higher rates of hypoglycemia without an improvement in survival rate. The review by Kramer and colleagues in this issue of Critical Care confirms these data but provides solid evidence about the relationship between hyperglycemia and worsened neurological outcome after acute brain injury. In accordance with the conclusions of Kramer and colleagues, we recommend that a glucose control goal in neurocritical care patients be in the 'moderate' target range (110 to $180 \mathrm{mg} / \mathrm{dL}$ ). In addition, we recommend adequate nutrition before and during insulin infusion, avoidance of insulin as a bolus, and the use of continuous insulin infusion, beginning with low doses with titration to individual sensitivity. Careful and accurate glycemic monitoring is especially important when insulin is infused.
\end{abstract}

The review and meta-analysis focusing on optimal glycemic control in neurocritical care (NCC) patients by Kramer and colleagues [1] in this issue of Critical Care provide a contemporary and comprehensive overview of randomized controlled trials (RCTs) that apply different glycemic control strategies in this challenging intensive care unit (ICU) population. Optimal glycemic control in critical care (CC) patients, in general, and NCC patients, in particular, has evolved dramatically over the past 15 years and remains under active investigation and debate

${ }^{+}$Contributed equally

*Correspondence: bilotta@tiscali.it

Department of Anesthesiology, Critical Care and Pain Medicine, Section of Neuroanesthesia and Neurocritical Care, 'Sapienza' University of Rome, Viale del Policlinico 1, Rome 00161, Italy about the ideal target range and impact of dysglycemia (high, low, and variable glucose levels) on outcome in the heterogeneous ICU population [2]. Prior to 2001, clinicians frequently applied a somewhat 'permissive' glycemic control approach in CC and NCC patients since hyperglycemia was considered a physiological response to stress or insult. Starting about a decade ago, the target range for glycemic control was the achievement of euglycemia ( 80 to $110 \mathrm{mg} / \mathrm{dL}$ ) by using intense insulin therapy as an infusion in the hopes of reducing ICU and hospital morbidity and mortality [3]. Subsequently, the latter approach was shown to result in an increased, but varying, incidence of hypoglycemia [4]. The incidence appears to vary by study, patient population, glucose goal, and intensity of insulin infused. However, hypoglycemia develops also in some critically ill patients in the absence of infused insulin. Whether there is an unfavorable cause-and-effect impact on outcome in critically ill patients who become hypoglycemic remains under scrutiny.

Currently, a key issue with tight glycemic control (target range of 80 to $110 \mathrm{mg} / \mathrm{dL}$ ) achieved by using intensive insulin therapy in NCC patients is the report of higher rates of hypoglycemia and lack of benefit on outcome [5-7]. Kramer and colleagues confirm these data but provide solid evidence about the relationship between hyperglycemia and worsened neurological outcome after acute brain damage.

In their review and meta-analysis, the primary outcomes were mortality at 6 months and level of neurological recovery/function (according to the quantitative functional scale used by the individual study). Secondary outcomes included hypoglycemia (defined by different thresholds ranging from 40 to $80 \mathrm{mg} / \mathrm{dL}$ ), nosocomial pneumonia, and other nosocomial infections. Articles to be retrieved and reviewed were searched by using three criteria: intensive glycemic control, NCC, and clinical trials (only RCTs were considered eligible).

In total, 23 studies were analyzed. Primary pooled outcomes were extracted from 16 studies that involved 1,248 patients. Reported mortality was similar in patients treated with intensive and conventional glycemic targets 
(26\% versus $27 \%$, relative risk (RR) $0.99,95 \%$ confidence interval (CI) 0.83 to $1.17, P=0.89$ ). However, poor neurological outcome was less frequent in patients who received intensive glycemic management (58\% versus $68 \%$, RR $0.91,95 \%$ CI 0.84 to $1.00, P=0.04$ ). Hypoglycemia was markedly greater among treated patients (30\% versus $14 \%$, RR $3.10,95 \%$ CI 1.54 to $6.23, P=0.002$ ). No effects were detected in the incidence of nosocomial pneumonia, and other nosocomial infections were infrequently reported, and thus a conclusive statistical analysis was not possible.

The results of this review and meta-analysis confirm that tight blood glucose control in NCC patients does not reduce mortality but increases the rate of hypoglycemia. The new message from this review it that, in patients treated with an 'active' glucose control strategy with insulin infusion to maintain a blood glucose concentration (BGC) of less than $180 \mathrm{mg} / \mathrm{dL}$, in comparison with those in whom 'loose' glycemia control was allowed (BGC of greater than $200 \mathrm{mg} / \mathrm{dL}$ before the start of insulin infusion), there is an improvement in neurological outcome.

Of relevance, the authors reported that information on nutritional status and nutritional support was reported poorly in the original articles but that in most of the cases, tube feeding appeared to have been initiated as soon as possible' [1].

In conclusion, optimal glucose control in NCC patients should include an active therapeutic strategy. Tight blood glucose control (with BGC target range of 80 to $110 \mathrm{mg} / \mathrm{dL}$ ) exposes NCC patients to an increased risk of iatrogenic hypoglycemia. Patients with hypoglycemia (BGC values of less than $80 \mathrm{mg} / \mathrm{dL}$ ) have increased mortality and the potential for worsened long-term functional status. On the other hand, patients with acute brain damage and permissive hyperglycemia with BGC values of greater than $180 \mathrm{mg} / \mathrm{dL}$ have a worsened neurological outcome.

During the last decade, there have been dramatic changes in our attitude toward glycemic control in NCC patients. It is now clear that this physiological variable cannot be overlooked and deserves committed clinical attention that is based on what we have learned. We now know that the time frame for manipulation of BGC is not as strict as that for hemodynamic variables and that sudden changes in BGC are potentially as dangerous as extreme $\mathrm{BGC}$ values [8]. We know that intense insulin infusion used to control glucose must be monitored appropriately and administered along with adequate enteral or parenteral nutrition and that iatrogenic hypoglycemia must be avoided. We also know that currently available clinical experience and technology are often derived from chronic management of patients with diabetes mellitus but that the unique characteristics of
$\mathrm{CC}$ and NCC patients require new understandings and applications of technology.

Research areas that require further investigation include the following:

1. Assessment of the impact of glucose control differentiated by NCC-specific pathology. This requires more information on the impact of glucose homeostasis and control in specific NCC patient subgroups: traumatic brain injury, ischemic/ hemorrhagic stroke, neuro-oncologic pathology, subarachnoid hemorrhage, and brain injury severity [911]. Of note is the study by Schlenk and colleagues [12], who used brain microdialysis in patients with subarachnoid hemorrhage to demonstrate the association of a target BGC of less than $110 \mathrm{mg} / \mathrm{dL}$ with increased acute brain metabolic derangements.

2. Mathematical models to describe and tailor an individual patient's glucose sensitivity, the changes in glucose sensitivity over time and the relationship between changes in insulin sensitivity and the insulin/ nutrition infusion protocol (amount of calories, access used for nutrition, amount and timing of insulin infusion) used in the individual patient.

3. Dedicated engineering technology, including continuous glucose monitoring devices with the application of closed loop insulin/nutrition infusion systems [13-15].

4. Greater understanding of the relationship between peripherally measured glucose, glucose in the healthy brain, and glucose in the injured or ischemic brain.

In accordance with the conclusions of Kramer and colleagues, we recommend that insulin infusion for glucose control in NCC patients be aimed at a 'moderate' target range (110 to $180 \mathrm{mg} / \mathrm{dL}$ ). In addition, we would recommend an adequate nutrition support of NCC patients before and during insulin infusion, the avoidance of insulin boluses, and the use of continuous insulin infusions initially at low dose, titrated to individual sensitivity with the application of a standardized and easily applied glycemic monitoring protocol.

\section{Abbreviations}

BGC, blood glucose concentration; CC, critical care; $\mathrm{Cl}$, confidence interval; ICU, intensive care unit; NCC, neurocritical care; RCT, randomized controlled trial; $\mathrm{RR}$, relative risk.

\section{Competing interests}

The authors declare that they have no competing interests.

\section{Acknowledgments}

The authors are grateful to Douglas Coursin for his thoughtful suggestions.

Published: 30 October 2012

\section{References}

1. Kramer AH, Roberts DJ, Zygun DA: Optimal glycemic control in neurocritical care patients: a systematic review and meta-analysis. Crit Care 2012, 16:R203.

2. Bilotta F, Rosa G: Glycemia management in critical care patients. World J Diabetes 2012, 3:130-134. 
3. van den Berghe G, Wouters P, Weekers F, Verwaest C, Bruyninckx F, Schetz M, Vlasselaers D, Ferdinande P, Lauwers P, Bouillon R: Intensive insulin therapy in critical ill patients. N Engl J Med 2001, 345:1359-1367.

4. Bilotta F, Caramia R, Paoloni FP, Delfini R, Rosa G: Safety and efficacy of intensive insulin therapy in critical neurosurgical patients. Anesthesiology 2009, 110:611-619.

5. Zafar SN, lqbal A, Farez MF, Kamatkar S, de Moya MA: Intensive insulin therapy in brain injury: a meta-analysis. J Neurotrauma 2011, 28:1307-1317.

6. Bilotta F, Giovannini F, Caramia R, Rosa G: Glycemia management in neurocritical care patients: a review. J Neurosurg Anesthesiol 2009, 21:2-9.

7. Bilotta F, Rosa G: Glucose management in the neurosurgical patient: are we yet any closer? Curr Opin Anaesthesiol 2010, 23:539-543.

8. Lundelin K, Vigil L, Bua S, Gomez-Mestre I, Honrubia T, Varela M: Differences in complexity of glycemic profile in survivors and non-survivors in an intensive care unit: a pilot study. Crit Care Med 2010, 38:849-854.

9. McCormick M, Hadley D, McLean JR, Macfarlane JA, Condon B, Muir KW Randomized, controlled trial of insulin for acute poststroke hyperglycemia. Ann Neurol 2010, 67:570-578.

10. Bilotta F, Caramia R, Cernak I, Paoloni FP, Doronzio A, Cuzzone V, Santoro A, Rosa G: Intensive insulin therapy after severe traumatic brain injury: a randomized clinical trial. Neurocrit Care 2008, 9:159-166.

11. Bilotta F, Spinelli A, Giovannini F, Doronzio A, Delfini R, Rosa G: The effect of intensive insulin therapy on infection rate, vasospasm, neurologic outcome, and mortality in neurointensive care unit after intracranial aneurysm clipping in patients with acute subarachnoid hemorrhage: a randomized prospective pilot trial. J Neurosurg Anesthesiol 2007, 19:156-160

12. Schlenk F, Vajkoczy P, Sarrafzadeh A: Impatient hyperglycemia following aneurysmal subarachnoid hemorrhage: relation to cerebral metabolism and outcome. Neurocrit Care 2009, 11:56-63.

13. Rice MJ, Coursin DB: Continuous measurement of glucose: facts and challenges. Anesthesiology 2012, 116:199-204.

14. Bilotta F, Doronzio A, Stazi E, Titi L, Zeppa IO, Cianchi A, Rosa G, Paoloni FP, Bergese S, Asouhidou I, loannou P, Abramovich AE, Spinelli A, Delphins E, Ayrian E, Zelman V, Lumbs P: All glucose measurements are not equal. Anesthesiology 2009, 111:1160-1161.

15. Skjaervold NK, Solligard E, Hjelme DR, Aadahl P: Continuous measurement of blood glucose: validation of a new intravascular sensor. Anesthesiology 2011, 114:120-125.

doi:10.1186/cc11521

Cite this article as: Bilotta F, Rosa G: Optimal glycemic control in neurocritical care patients. Critical Care 2012, 16:163. 